\title{
Téoros
}

Revue de recherche en tourisme

\section{Une région de prédilection pour des établissements de tourisme social}

\section{Les centres de vacances}

\section{Louis Jolin}

Volume 15, numéro 1, printemps 1996

Les Laurentides, quelles Laurentides?

URI : https://id.erudit.org/iderudit/1075049ar

DOI : https://doi.org/10.7202/1075049ar

Aller au sommaire du numéro

Éditeur(s)

Université du Québec à Montréal

ISSN

0712-8657 (imprimé)

1923-2705 (numérique)

Découvrir la revue

Citer cet article

Jolin, L. (1996). Une région de prédilection pour des établissements de tourisme social : les centres de vacances. Téoros, 15(1), 23-25.

https://doi.org/10.7202/1075049ar d'utilisation que vous pouvez consulter en ligne.

https://apropos.erudit.org/fr/usagers/politique-dutilisation/ 


\section{UNE RÉGION DE PRÉDILECTION POUR DES ÉTABLISSEMENTS DE TOURISME SOCIAL LES CENTRES DE VACANCES

\author{
Louis Jolin
}

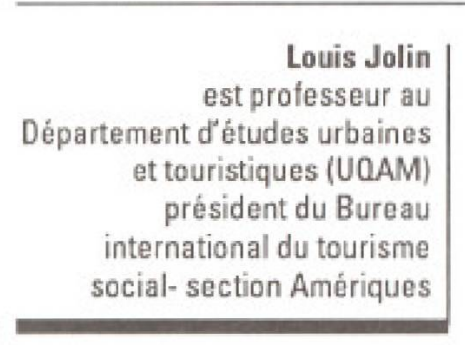

Sı la grande réglon des Laurentides (Incluant les régions touristiques des Laurentldes, de Lanaudière et une partle de l'Outaouals) a accuelli, au fll des ans, des établlssements prestigleux qui ont fait sa réputation au plan International pour la qualité de leur table ou de leur hospitalité, elle a vu ausst croître une foule d'établissements qui ont comme mIssion de favoriser la découverte de la nature et la pratique d'activités de plein alr et de lolsir touristique. II n'est pas question d'opposer ici ces établlssements même si les objectifs poursulvis par les uns et par les autres ne sont pas IdentIques: l'auberge du P'tlt Bonheur a une réputation comparable à La Saplnlère mals sous un autre registre.

\section{Les centres de vacances}

Région à proximité de celle de Montréal, la grande région des Laurentides a répondu et répond toujours aux besoins sociaux tels que ressentls dans les divers quartiers de la métropole: besolns de vacances en nature pour les leunes. pour les familles, pour les aînés, pour les personnes à capacité physique restreinte, besoins de ressourcement et d'aération après de longs mois d"une vie urbalne stressante. Tous ces besolns sont au coeur de l'action de plusleurs organismes ou associations qui tentent d'offrir une réponse concrète, c'est-âa-dire un lieu d'accucll, des actlvités adaptées, des prix abordables. Cette réponse se décline sous diverses appellations: camps ou colonles de vacances pour jeunes, camps familiaux, bases et centres de plein air, camps spéclalisés... Le règlement d'appllcation de la Lol sur les établissements touristiques établit la sous-catégorie centre de vacances pour recouvrir cette réallté multiforme ${ }^{1}$. Une grosse majorlté des centres de vacances sont de véritables équipements de tourisme social dans la mesure où Ils accuellent prloritairement des groupes de population qui pourraient difficilement partir en vacances autrement pour des ralsons financlères, pour des ralsons de santé ou autres. A but non lucratif, Ils constituent le noyau dur du tourlsme soclal, expression et phénomène qul ne se résument pas cependant à cette seule dimension, aussi essentielle solt-elle à lcur compréhension.

\section{La diversité du tourisme social}

Le tourisme soclal embrasse une sérle de réallsations exprlmant l'ambition de vacances pour tous et la volonté d'étendre la pratlque du tourisme à toutes les couches de la soclété: ces réallsations veulent aussi contribuer au développement socloeconomlque des usagers comme des milleux daccuell, avec le soucl de proposer des actlvités à caractère éducatif et culturel. en valorlsant le pa t r I m o I n e naturel ou culturel. Le tourIsme soclal intègre des "objectlfs humanistes. pédagogiques, culturels, d'insertion, de promotion et d'épanouissement des individus, ${ }^{\text {" }}$

Les parcs nationaux, provinclaux et régionaux, les centres d'Interprétation de la nature, les écomusées, le phénomène de l'agrotourisme sout autant de réallsations non exclusives à l'enseigne d'un tourisme dont on accole l'adjectif soclal pour souligner que l'objectif n'est pas la recherche d'un profit maximal (ce qul ne veut pas dire que les réallsations ne dolvent pas tendre vers la rentablité et adopter des méthodes de gestion efficaces ${ }^{3}$ ). mals plutôt d'apporter un surplus social, une plus-value sociale (contribuer à la cohésion soclale et à l'épanouissement culturel, social et économlque des individus et des collectivités, lutter contre l'exclusion. promouvoir et valoriser le patrimoine naturel et culturel. etc.). 


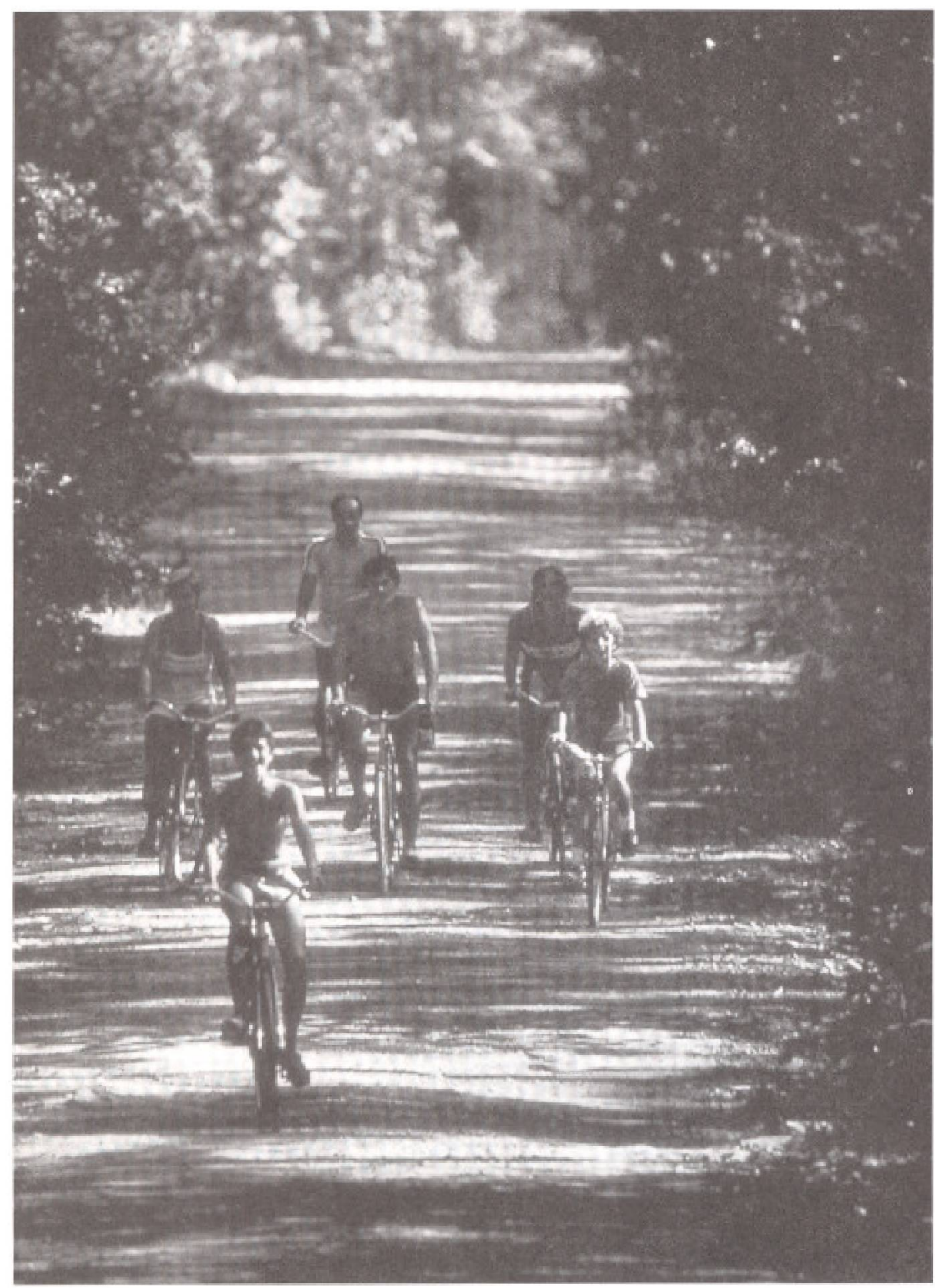

locaux dans l'animation et la gestion de ces établissements.

Lorsqu"on consulte le dernler répertolre de l'Association des camps du Québec ${ }^{4}$. sur 99 camps de vacances accrédités (excluant les camps de jour), 24 sont sltués dans la réglon touristique des Laurentides, 16 dans la réglon tourlstlque de Lanaudière, sans compter les quelques camps de l'Outaouals que l'on peut ralsonnablement Intégrer dans la grande réglon géographlque des Laurentides 5 . Près de $45 \%$ des camps de vacances accrédités de l'Assoclation des camps du Québec se retouvent donc dans la grande réglon géographlque des Laurentides. Parml ces camps, certains y sont établls depuls de très nombreuses années: le Camp Oolahwan a ếté fondé en 1917. le Camp Weredale en 1934, le Camp Bruchésl en 1936. la Base de plein air Jean-Jeune depuls 50 ans comme le Camp Marcel (malntenant désigné sous le nom d'Havre famlllal, centre de plein alr), etc.

Camps francophones et anglophones, Ils reflètent blen la dualité lingulstique de la métropole comme de la réglon des Laurentides elle-même. Parml les camps qui déterminent le coût du séfour selon le revenu familial (11), plus de $70 \%$ sont sltués dans cette réglon. Cette façon de fixer le coût du séfour révèle un soucl de tenir compte des contraintes financières des familles et s"inscrit dans une vlellle tradition du tourisme soclal en vole de disparition. Enfin de nombreux camps ou colonies de vacances ont effectué des modiflcations dans leurs modes de fonctionnement afin de rejolndre, à l'année, une cllentète plus diversiflée. avec une programmation au goût du jour (activités hivernales, cours de langue.

\section{La région des Laurendides: région de prédilection}

Plusleurs réglons du Québec ont des équipements de tourlsme soclal, mals la grande réglon des Laurendides se démarque particulièrement par certains types d'établissements, notamment les centres de vacances. Il ne s'aglt pas nécessalrement d'un cholx délibéré des acteurs réglonaux qui auralent cholsl ce type de développement plutót qu'un autre (au contraire. les centres de villéglature, les centres de skt ont eu la faveur), mals cest le résultat des inttlatives d'organismes du milieu métropolitain, oeuvrant dans un grand bassin de population à proximité de la réglon (groupes religleux au début, associations communautalres, YMCA, centres de lolsirs, groupes familiaux, etc.). La réglon en a pris acte. parfols à son corps défendant, bien que nous assistions plus qu'autrefols à l'implication des résidents séances d'initiation à l'informatique en plus des activités sportives et de plein air). Ces modifications poursulvent un double objectif: rendre l'offre de services plus attrayante compte tenu des changements dans les habitudes de consommation et assurer un melleur financement du fonctionnement et des immobilisations pour parer aux balsses de subventions des pouvoirs publics.

Le répertoire des camps familiaux ${ }^{6}$, qui recense les groupes et les camps membres du Mouvement québécols des camps famillaux et visant à offrìr aux familles aux revenus modestes des vacances épanoulssantes et accessibles, réflète également cette même concentration dans la grande réglon des Laurentides. Si les groupes famillaux comme tels se retrouvent à plus de $55 \%$ sur liile de Montréal, les établissements (camps lamillaux) sont situés pour la moltié d'entre eux dans la grande région des Laurentides. 


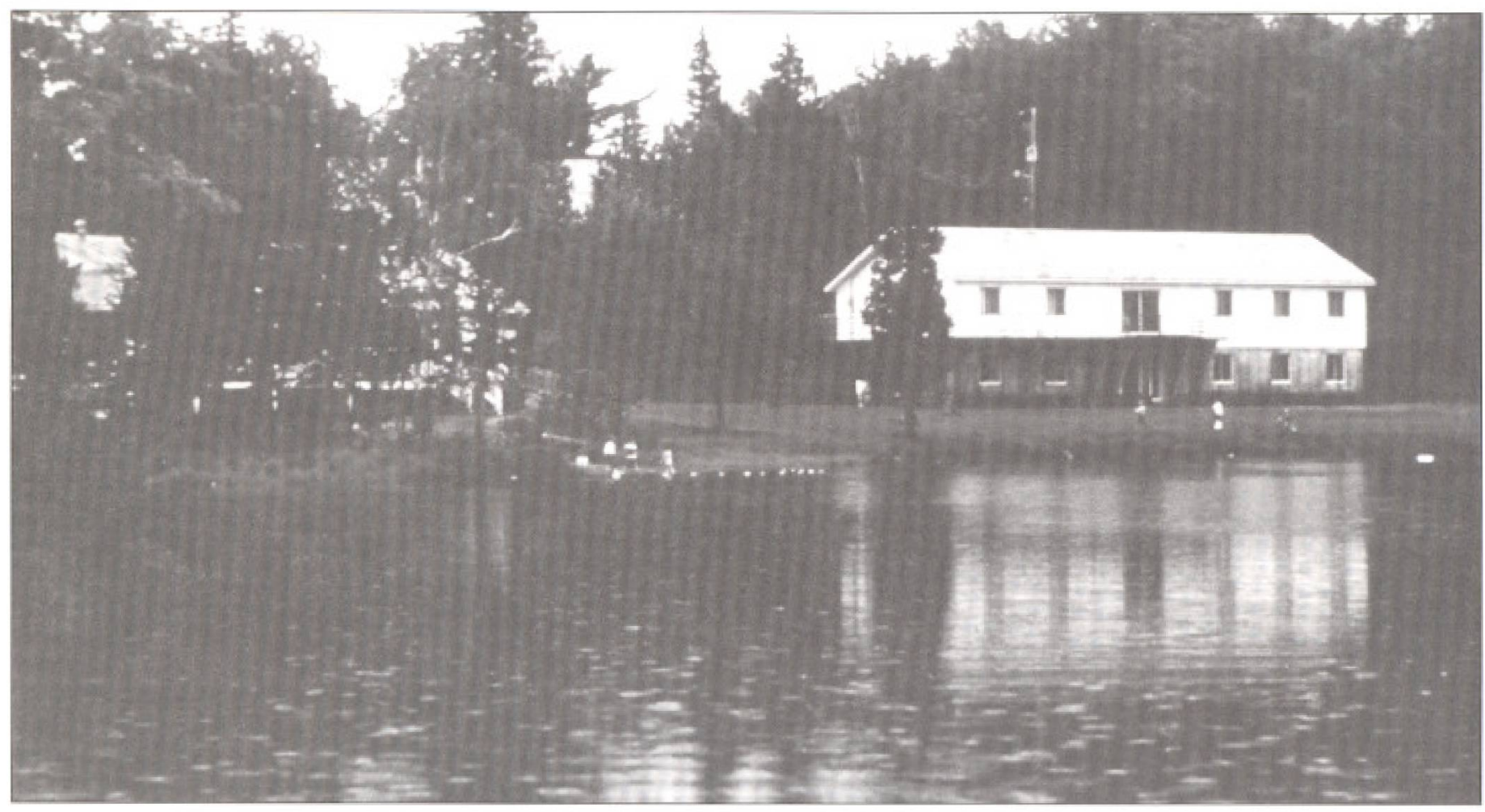

\section{Un apport méconnu}

Pour les Montréalais, cette région n'est-elle pas la cour de récréation par excellence pour des excursions de dimanche apres-midi, une soirce ou un week-end de ski? S'il est vrai que les Montréalais l'ont adoptée pour sa proximité comme pour ses attraits (montagnes, lacs, forêts, villages, parcs, campings, centres de ski...). des organismes sociaux, des associations de loisir et de tourisme y ont installé des centres de vacances pour les mêmes raisons. Cet apport pour la région est loin d'être marginal mais n'est pas toujours reconnu par ses habitants et ses édiles. La contribution de ces établissements dans l'aménagement du territoire est souvent méconnu. Leurs retombées sociales et économiques insufisamment soulignées7. Les causes de cet état de fait sont multiples ed tiennent à la fois à la compréhension que l'on se fait de l'activité touristique et des priorités que l'on se fixe comme région touristique, mais aussi du peu d'empressement de plusieurs responsables de centres de vacances à insérer leur projet dans la dynamique et la mouvance régionales.

Mais quol qu'on en dise, les centres de vacances ont néanmoins une REGION! votes

1. "La sousucategorie "centwe de vacanees" regroupe les établissements qui offrent an public, moyennamt un prix forlatiaire, de lhebergement det des set=

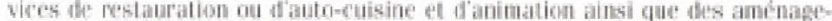

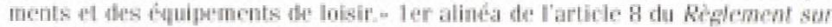

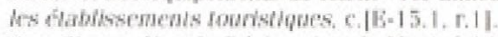

2 Tus Singer. fers fa beclarabon de Wontreal focument de consulation en vue to l'assemblée aenerale du bl's de septembre 1996). Bruxelles, Bareau intermatichal du tourisme social, decembre 1995, p. 15

3 ela perlormance bconomique est la comdition mèn de la permanence du proiet.- Itrid. . 10. 3.

4 Association des camps du Oudiec, kepervore - Camps do cacances el camps the Marr. Montréall. edition 1996.

5 Ia deuvième région tă la grande répion de ouebec avee 10 átablissements

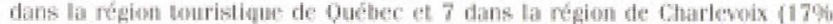
des camps accrídites de l'Associatin des camps du ouébec sans compher les calips de jour).

6) Mouvement québécois des canps familiaux, Les camps hamilan - Répervoire

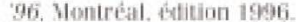

7 On commence à psine à cerner l'importance économique des centres de vacancrs. line récente recherche a permis d'evaluer à 57214 le nombre de

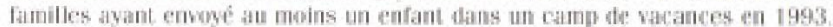
le nombre moyen denfants par famille dans les camps au cours de cette dannée est de 1,15 , le durée moyenne de sćfour dans un camp est de 11.4 jours. st le montant dépensé par jour-ramperur est de $27 \$$, se qui représente un marché total des camps d'été pour les frunes de 202520528 Source- Zins Batchesse et associés, Rapport final présenté à lassociation des camps du Quebec et au minsitere du Loisir, de la Chasse ell de la Pieche. 1994. 